\title{
El arte como visibilizador de complejidades sociales / la complejidad social como invisibilizadora del arte
}

\section{Art as a visualizer of social complexities / social complexity as an invisibilizer of art}

TIPO DE TRABAJO: Comunicación.

\author{
PALABRAS CLAVE
}

Arte sistémico, productivismo, arte y espacio social, gestión de la complejidad, visibilidad-invisibilidad.

KEY WORDS

Systemic art, productivism, art and social space, complexity management, visibility-invisibility.

RESUMEN

En el contexto actual algunas de las prácticas artísticas se caracterizan por una tendencia a la desmaterialización y a la concepción de la obra-como-proceso, actitud que contribuye a mitigar su presencia y notoriedad en un contexto marcado por la hipervisibilidad y la inmediatez. Ante la necesidad de hacer inteligible la complejidad social, en un contexto de sobrexposición, el arte puede ser una forma de acción que contribuya a visibilizar la realidad e incidir en ella. Pero si el arte no consigue regenerar y pluralizar sus mecanismos de producción, puede quedar excluido de esta labor socializadora. La producción artística, entendida desde una perspectiva sistémica, puede afrontar la gestión de la complejidad como esencia, a la vez que conectarse a otros ámbitos, disciplinas o formas de ver-entender el mundo, que comparten el objetivo de descifrar e investigar para trazar futuros posibles.

Desde lo productivo, el arte se inscribe en un ecosistema propio compuesto por ámbitos ocasionalmente interconectados. Uno de ellos es el mercado del arte, enraizado en una economía pre-fordista, que consiste en la venta de productos "exclusivos" a clientes "exclusivos". Otro es el modelo promovido por la acción de los poderes públicos, con el objetivo de impulsar la democratización, difusión y creación cultural. Un planteamiento de tradición malrauxista, en el que las instituciones públicas dotan a la cultura un papel vinculante y una partida económica en la tarea de contribuir a modernizar socialmente el país. Un tercer modelo de carácter híbrido y experimental, estaría caracterizado por el ensamblaje de estrategias productivas diversas, desde la autogestión, a la incursión en economías colaborativas, la creación conectada a espacios intersticiales entre el espacio artístico y el social, o la mezcla de todo ello con financiación pública. Desde esta tercera vía se exploran elementos relacionados con su visibilidad e invisibilidad.

\section{ABSTRACT}

In current context some of the artistic practices are characterized by a tendency towards dematerialization and the conception of the work-as-process, an attitude that contributes to mitigate its presence and notoriety in a context characterized by hypervisibility and immediacy. Given the need to make intelligible the social complexity, in a context of overexposure, art can be a form of action that contributes to visualize and influence reality. But if art fails to regenerate and pluralize its mechanisms of production, it can be excluded from this socializing achieve. Artistic production, understood from a systemic perspective, can face the management of complexity as essence, while connecting to other areas, disciplines or ways of seeing-understanding the world, which share the goal of deciphering and investigating to trace possible futures.

From the productive, the art is part of an own ecosystem of occasionally interconnected areas. One of them is the art market, rooted in a pre-Fordist economy, which consists of the sale of "exclusive" products to "exclusive" clients. Another is the model promoted by the action of public authorities, with the aim of promoting democratization, dissemination and cultural creation. An approach of 
malrauxist tradition, in which public institutions endows culture with a binding role and an economic contribution in the task of contributing to the social modernization of the country. A third model of hybrid and experimental character, would be characterized by the assembly of diverse productive strategies, from self-management, to the incursion in collaborative economies, the creation connected to interstitial spaces between the artistic and social space, or the mixture of them, with public funding. From this third way, elements related to its visibility and invisibility are explored.

\section{INTRODUCCIÓN}

En el contexto actual algunas de las prácticas artísticas se caracterizan por una tendencia a la desmaterialización y a la concepción de la obra-como-proceso, actitud que contribuye a mitigar su presencia y notoriedad en un contexto marcado por la hipervisibilidad y la inmediatez, también por la hiperinformación. Byung-Chul Han nos alerta de este exceso de información que contribuye a hacer un mundo más confuso "la hiperinformación y la hipercomunicación no inyecta ninguna luz en la oscuridad" (p. 80).

Una de las funciones que tradicionalmente ha cubierto la cultura (y de forma muy concreta el arte) está asociada a la idea de visibilizar la percepción del mundo, e introducir un ámbito de experimentación y análisis crítico en relación con el espacio social. En estos momentos aparecen nuevos actores en la gestión de las políticas culturales que, influenciados por las estrategias neoliberales, plantean unas programaciones derivadas de las necesidades del mercado, interesados en incrementar los públicos, convirtiendo las actividades culturales en espacios de entretenimiento y espectáculo mediante el fomento del turismo cultural, el generar actividades sucintas a demanda o su inserción en el sector de las industrias culturales. Esto plantea un cambio importante en la relación entre las políticas culturales y la producción artística.

Ante la necesidad de hacer inteligible la complejidad social, en un contexto de sobrexposición, el arte puede ser una forma de acción que contribuya a visibilizar la realidad e incidir en ella. Pero si el arte no consigue regenerar y pluralizar sus mecanismos de producción, puede quedar excluido de esta labor socializadora. La producción artística, entendida desde una perspectiva sistémica, puede afrontar la gestión de la complejidad como esencia, a la vez que conectarse a otros ámbitos, disciplinas o formas de verentender el mundo, que comparten el objetivo de investigar, descifrar y comunicar para trazar futuros posibles.

\section{METODOLOGÍA}

Esta comunicación se ha redactado a partir de una metodología de investigación fundamentada en el cruce de diferentes referentes bibliográficos, relacionados con el tema planteado. Cabe decir que la temática es fruto de una experimentación previa, trazada a partir de años de implicación personal en desplegar una práctica artística fundamentada en plataformas de producción vinculadas a contextos específicos, a una voluntad de insertarse o construir espacios sociales y una preocupación por dar visibilidad a estas formas de producción. Este tipo de trabajo de naturaleza sistémica, trata de buscar en este y otros textos, una forma de teorizar o dar sentido a una forma de entender la investigación artística fundamentada en lo experimental y experiencial.

\section{DESARROLLO}

\section{1. ¿Cual es la funcionalidad visible del arte?}

Algunas de las habituales preguntas que debe encajar el arte están relacionadas con su presencia, visibilidad y función: ¿qué sentido tiene? y ¿para qué sirve?. Cuestionando el motivo de su existencia y su utilidad, se apela al profundo distanciamiento existente entre las prácticas artísticas contemporáneas y las relaciones de producción y distribución en el contexto del capitalismo contemporáneo; donde la producción material o inmaterial es fruto de una demanda relacionada con algún tipo de carencia (o necesidad) identificable, sea subjetiva o social, una difusión publicitaria que contribuye a seducir y estimular el deseo subjetivo de consumo y un flujo comercial vinculada a ella. La clásica regla de la producción que se ciñe a la oferta y la demanda, nunca ha tenido mucho sentido en la producción artística. En la economía capitalista su valor no responde al valor de uso, si no al valor de cambio que se construye a partir de una compleja trama de intermediarios, coleccionistas, museos, ferias de arte y bienales. Mientras que el arte en el pasado era celebrado por sus efectos catárticos, su religiosidad, su pedagogía moral o solidaridad revolucionaria, el arte contemporáneo recibe el mandato de ser crítico (Squibb, 2017, p.23). La producción del discurso crítico ha constituido una de las materias primas más fructíferas en la producción artística contemporánea, distribuida y consumida en el circuito internacional, aún cuando este discurso cuestione reiteradamente cada una de las partes que alimentan el sistema globalizado del arte. 
Esta proliferación de la crítica institucional ha contribuido a la desmaterialización de la obra y a la incorporación de los espacios del arte como una formalización visible de una materia invisible. La producción de acontecimientos estéticos es una de las características del arte contemporáneo, según Boris Groys (2016), que no pueden ni deben presentarse como obras tradicionales de arte. Mientras el arte tradicional produce objetos de arte, el arte contemporáneo produce información sobre acontecimientos de arte. Es en este sentido que los museos e instituciones concebidas desde una visión tradicional del arte no pueden cubrir las necesidades de algunas de las formas de creación artística contemporánea, en especial aquellas que se articulan desde la construcción colectiva, la incorporación participativa de otros agentes involucrados, o aquellas formas más complejas que ensamblan distintas prácticas procesuales. La proliferación de trabajos fundamentados en la participación y colaboración plantean nuevos problemas en relación a su puesta en escena y su consecuente visibilidad. En este sentido Hal Foster habla de una "promiscuidad de colaboraciones" en un texto donde problematiza los conceptos de participación y colaboración en el arte contemporáneo, por el hecho de que se genera una "promiscuidad de instalaciones" que son las que pueden verse en las múltiples bienales (Foster, H. 2006, p. 190-195). A su juicio, estas instalaciones aglutinan gran cantidad de textos, vídeos, objetos, ocasionando un efecto más caótico que comunicativo. El arte que se produce en estas circunstancias plantea, en muchos casos, problemas de visibilidad y comunicación en los tradicionales espacios de representación. En este tipo de trabajos adquieren importancia elementos como las discusiones, los encuentros, las vivencias, los acuerdos, etc., todos ellos parte imprescindible del proceso de socialización creativa, constituyendo en muchos casos la propia obra.

Estos procesos no son fácilmente traducibles en los límites del espacio expositivo, y esto genera una tensión crítica en las instituciones artísticas, aún fundamentadas en el formato más clásico de exhibición. Para resolver estos problemas de accesibilidad, según Groys (2016), "hay que volver fluida la forma de la obra" (p.21), refiriéndose con ello en multiplicar los formatos de distribución, socialización y publicación. Los proyectos basados en procesos colaborativos y participativos no pueden analizarse desde la lógica de la habitual puesta en escena. Forman parte de un territorio de transversalidad que activa nuevas prácticas culturales, las cuales podrán incidir de manera activa en el contexto social. Estas formas de producción artística que desbordan la lógica del objeto atemporal del arte, encuentran en el concepto "modernidad líquida" (Bauman, Z. 2000), una buena interpretación acerca de un tipo de prácticas de carácter temporal, vinculadas a contextos y circunstancias específicas, desmaterializadas, de autorías difusas, de composición híbrida y generadas en una red globalizada. El adjetivo "líquido" refleja los efectos de la globalización, el turismo, las migraciones, el nomadismo, la comunicación global, el mercado sin fronteras, la flexibilidad laboral. A pesar de que la condición "líquida" se adapta bien a este tipo de prácticas artísticas, Bauman parece no querer entender que también el arte es fruto de las circunstancias de estos tiempos que tan bien ha descrito: "Ias obras de arte no son 'útiles', 'funcionales'; no sirven para asegurar la supervivencia del individuo. Su inmortalidad radica en que se alejan del metabolismo de la vida" (Bauman, Z. 2007, p.17). De manera divergente a esta consideración del arte, aquí nos posicionamos y ponemos el foco hacia un tipo de prácticas que buscan conectarse con la vida, que abordan la complejidad de lo social y afrontan el problema de la visibilidad en un enmarañado contexto de hipervisibilidad y de comunicación fugaz. Nos preocupa como multiplicar las funcionalidades de las prácticas artísticas.

Uno de los argumentos para justificar el grado de visibilidad y utilidad social que tiene el arte es recurrir a la idea de el arte como herramienta. Herramienta para ayudar a las personas a lidiar con la vida y la condición humana, herramienta de mediación en contextos sociales desfavorecidos, herramienta educativa, herramienta que puede ayudar a mejora la calidad de vida de las personas, herramienta para facilitar la cohesión social, herramienta que otorga una carga simbólica a un determinado contexto, herramienta de comunicación, de propaganda, de innovación, de visibilidad, de participación, de acción directa, herramienta que incentiva el espíritu crítico o el goce estético. Su utilidad se multiplica y la funcionalidad se diversifica. La práctica artística como herramienta y en un contexto de creación colectiva, no ejerce una única función, la utilidad se genera en relación con el sujeto, la comunidad, la situación concreta, los objetivos que se persiguen o los agentes que participan en la acción creativa. Esta idea de utilidad social o fusión del arte con la vida cotidiana, o compromiso con el contexto social, ha formado parte de la problemática abordada por alguna vanguardia artística de principios del siglo XX, y des de finales de los años 60 aparece de forma problematizada y recurrente hasta la actualidad. Por ejemplo la artista Tania Bruguera ha propuesto el concepto Arte Útil, que trata sobre como debería ser un tipo de arte que se implique en su uso social. Conjuntamente con el colectivo Democracia recogían en la publicación Nolens Volens 5 algunos aspectos y ejemplos de este planteamiento para remarcar la potencialidad del "arte útil", crear nuevas vías de relación entre arte y sociedad, construyendo alianzas con movimientos que aspiran a ser transformadores. También para tratar sus contradicciones, "como la instrumentalización a las que este tipo de proyectos pueden verse sometidos cuando se convierten en sucedáneos de verdaderos cambios sociales"1.

En el contexto contemporáneo de sobreexposición, las prácticas artísticas que se caracterizan por una tendencia a la desmaterialización, a la concepción de la obra-como-proceso y a la configuración de procesos colectivos, plantean alternativas a las condiciones fundamentadas en la producción subjetiva del arte, así como buscan otras formas para materializarse. En este sentido, desde el sistema económico que rige la distribución y comercialización del arte, este tipo de prácticas se generan en el marco de las instituciones públicas o programas independientes, que habitualmente financian la producción, mediante proyectos, encargos, comisariados o residencias artísticas; y sus resultados se hacen visibles en exposiciones temporales, publicaciones, eventos 0 manifestaciones puntuales. Aunque el mercado del arte puede incorporar cualquiera de estos trabajos (y en muchos casos así ocurre), su espíritu se forja en una lógica distinta a la de aquellos trabajos pensados para ser coleccionados y archivados, estos otros se

\footnotetext{
${ }^{1}$ Bruguera, T.; Democracia (2011). Arte Útil (en línea).
} 
fundamentan no tanto en el producto como en su función social. Las instituciones culturales en nuestro contexto, han seguido un planteamiento de tradición malrauxista, en el que dotan a la cultura un papel vinculante y una partida económica en la tarea de contribuir a modernizar socialmente el país. Esta idea de democratizar la cultura e implicarse en la transformación social, ha configurado un espacio de posibilidad creativa y de ensayo de otras formas de crear vinculadas al espacio social. Aunque en el contexto contemporáneo donde la política sigue una trayectoria de liberalización, privatización y transferencia de poder a otros espacios de poder privatizados, este planteamiento afronta aspectos de fragilidad y de dudosa continuidad.

\section{Desde el productivismo}

Para ampliar el campo de visión en relación a la producción artística contemporánea que persigue una voluntad de implicarse en lo social y plantea nuevas formas de visibilidad, vamos a hacer referencia a uno de los movimientos de vanguardia ruso soviética como es el productivismo². Encontramos en él una exploración de la politicidad del arte en el deseo de abrazar procesos de transformación social profundos (Expósito, M. 2007). El productivismo, supuso una propuesta inserta en un proceso social disruptivo, que introducía un replanteamiento y una lucha contra la autonomía y subjetividad de la práctica artística, contra cualquier tipo de estilización de épocas pasadas, contra todo tipo de ornamentación o embellecimiento de las formas, con la voluntad de integrar el arte al servicio de la mejora de la producción, poniendo la práctica artística al servicio de la colectividad, en el contexto de una sociedad socializada. El productivismo es una corriente teórica social, más que estética, sobre la producción artística. A pesar de ello, las relaciones entre forma y contenido fueron una de las preocupaciones tratadas por su máximo exponente teórico, Borís Aravátov. Mientras que los formalistas pretendían estetizar la utopía, el productivismo se consideraba como la utopía materializada. El productivismo ponía el interés en la cultura material entendida desde la conexión entre producción y consumo, en una nueva sociedad sin lucha de clases, donde se instauraba una alternativa a la lógica capitalista -donde la clase trabajadora produce y las clases altas o burguesas consumen-. El interés del productivismo pasaba por conectar producción, distribución y consumo en el seno de la vida cotidiana, construyendo ideología y, desde la práctica colectiva poder transformar la esfera social. Constituyó una forma de entender el arte integrado en la producción. Esto le otorgaba una razón de ser y una conexión con el concepto de utilidad desde una perspectiva social altamente politizada. En este sentido otro de sus deseos era desconectar el arte de la producción puramente formal para conectarla a la creación intelectual, y colaborar con otros colectivos para mejorar producción social (en aparatos estatales de propaganda o de cultura física, con asociaciones de periodistas, de lingüistas, con ingenieros, en las industrias o en las fábricas). Se planteaba que la funcionalidad técnico-social debía ser reconocida como la única ley y el único criterio de la actividad artística, en tanto que generadora de formas, donde el fetichismo de los procedimientos, las formas y la funciones estéticas de los materiales debían ser abolidos (Aravátov, B. 1926, p.109). El proyecto productivista trabajó para la colectivización del arte, para desprenderse de su formato tradicional y dotarlo de una nueva configuración, sumergiéndolo en el proceso de construcción colectiva de una utopía social. Las expresiones: “iAbajo el arte, viva la técnica!”, “iAbajo el arte, que solo enmascara la impotencia de la humanidad!” o “iEl arte colectivo del presente es la vida constructiva!", constituyen tres de las seis consignas expresadas en el manifiesto productivista. Son suficientemente claras para entender la propuesta de disolver el arte en un proceso de vida colectivo.

Para Rancière las prácticas artísticas son maneras de hacer que intervienen en la distribución general de las formas de hacer y sus relaciones con las maneras de ser y las formas de visibilidad. En la revolución rusa, arte y producción se identificaron; el culto del arte suponía una revalorización de las capacidades ligadas a la idea misma de trabajo, provenían de una misma repartición de lo sensible, fabricaban objetos a la vez que daban visibilidad (Rancière, J. 2014). El productivismo fue un experimento fallido en la época, de la misma manera que se puso al servicio del aparato político, y trabajó para contribuir a que la creatividad y el arte penetraran y se naturalizaran como forma de vida, como ingeniería social o transformación de vida cotidiana; el stalinismo lo fagocitó y lo incorporó al sistema, devolviendo al arte la estética perdida en forma de realismo socialista. Desde un planteamiento contemporáneo, y en relación al tipo de prácticas colaborativas que nos referimos, el productivismo nos aporta referencias históricas, marco teórico y experiencias concretas, para afrontar esta relación productiva entre lo social y lo político.

\section{Desde el arte sistémico}

El escultor y crítico de arte Jack Burnham, planteó en 1968 que el arte puede ser concebido y analizado como un sistema. La complejidad de componentes que configuran un sistema en el arte, desde el punto de vista del artista como creador y productor, puede contener personas, ideas, mensajes, condiciones atmosféricas, fuentes de energía, acciones educativas, mediaciones etc. La coherencia del sistema puede ser alterada en el tiempo o en el espacio, y su comportamiento determinado por las condiciones externas o los mecanismos de control, Burnham, J. (1968). Otro tema que introdujo es la visión de lo nuevo. Mientras que en muchos casos se plantea que la innovación es fruto de la creatividad artística (pensamiento heredero de la tradición de a autonomía del arte), él plantea que aquella está envuelta por las condiciones sociales y temporales, que son las que acaban valorando y condicionando la percepción de lo nuevo: "La innovación de las artes plásticas desde 1960, se sitúa más que nunca en el marco dialéctico de la relación estructura -

\footnotetext{
2 El Productivismo fue una de las vanguardias ruso soviéticas vinculadas al Constructivismo, que tuvo como teórico a Borís Aravátov, quien recogió las principales ideas en artículos como Arte y producción (1926). Según este autor, después de la revolución que dio pie a la Unión Soviética, un grupo de artistas y teóricos conocidos por el nombre de LEF: Lievi front iskusstva (Frente de Izquierdas para las Artes), asumieron el desarrollo del programa de arte productivista. En 1920, Alexander Rodchenko y Várvara Stepánova publicaron el Manifiesto Productivista.
} 
superestructura del sistema social del capitalismo tardío" (Ibíd. p.14). De todas formas, advierte que esta inserción de las artes con el sistema social no debe impedir el análisis específico de sus lenguajes en el marco de una autonomía relativa.

Entender la práctica artística como una articulación sistémica constituye un antecedente importante donde se concreta y argumenta por qué algunos de los trabajos que se realizaron a finales de la década de los 60 responden a esta idea sistémica que Burnham intuye, recoge y configura.

\section{CONCLUSIONES}

Aunque no hemos citado explícitamente casos concretos, las prácticas artísticas a las que nos hemos referido articulan procesos, estimulan formatos participativos y/o colaborativos, están interesadas en la interdisciplinariedad y activan estrategias de comunicación para conectar con nuevos públicos, o para buscar la implicación de la ciudadanía con el fin de diversificar el concepto de público. Son elementos, todos ellos, que pueden formar parte de una voluntad de reconectar la práctica artística con determinados espacios de lo social, definir funciones y estrategias de utilidad social y buscar otras formas de producción, presencia y visibilidad.

Cuando hablamos de prácticas artísticas colaborativas o participativas, se hace referencia a un ecosistema complejo, en el que el arte unas veces actúa de motor y se extralimita de sus dominios de legitimidad; y otras es vector de una noción de transversalidad, en el que actores y recursos propios del campo artístico se conectan con proyectos y experimentos que no se resuelven en el interior de dicho ámbito, sino que se expanden a otros lugares ${ }^{3}$. Es en este sentido que se quiere hacer referencia a un tipo de prácticas que, por el hecho de gestionar la complejidad tanto de sus componentes como de sus interacciones con lo que no le es propio, las denominamos prácticas artísticas sistémicas.

Hemos utilizado como referentes dos momentos históricos del pasado siglo, que nos sirven de contraste, nos aportan ideas y experiencias concretas, para afrontar la práctica artística desde la contemporaneidad. Aunque el contexto sociopolítico es completamente diferente, considero que hay ciertas relaciones conceptuales, cuando lo que buscamos son nuevos mecanismos y estrategias de producción, para dar visibilidad a la complejidad contemporánea y contribuir a aportar otras visiones frente a problemáticas, que desde una perspectiva tanto local como global, y desde el presente, afectan nuestros futuros posibles.

En este contexto de hipervisibilidad e hipercomunicación, son pertinentes estas palabras de Alain Badiou: "Estamos inmersos en una situación artística absolutamente confusa e incierta, porque se trata de un período de intervalo en el que las potencias del arte deberán ser reconstruidas según una modalidad infinitamente más afirmativa, infinitamente más ligada a los procesos reales, a las propuestas de la política" (p.106).

\section{FUENTES REFERENCIALES}

Arvátov, B. (2018). Arte y producción. Madrid: Ediciones Asimétricas.

Arvatov, B., y Kiaer, C. (1997). Everyday Life and the Culture of the Thing (Toward the Formulation of the Question). October, 81, 119128.

Badiou, A. (2013). La filosofía y el acontecimiento. Buenos Aires: Amorrortu editores.

Bauman, Z. (2000). Liquid modernity. Cambridge: Polity.

Bauman, Z. (2007). Arte, ¿líquido?. Madrid: Ediciones sequitur.

Burnham, J. (1968). System Esthetics. Artforum, 7.

Byung-Chul, H. (2013). La sociedad de la transparencia. Barcelona: Herder.

\footnotetext{
${ }^{3}$ Sobre el tema de la noción de transversalidad y la propuesta de una tercera fase de la crítica institucional, en la que se pretende superar el bucle sobre el que la crítica institucional previa, que se encierra en si misma, optando por una institucionalización de la crítica institucional; ver HOLMES, Brian (2007).
} 
Parramón Arimany, Ramón

El arte como visibilizador de complejidades sociales / la complejidad social como invisibilizadora del arte

IV Congreso INTERNACIONAL DE INVESTIGACIÓN EN ARTES VISUALES ANIAV 2019 IMAGEN [N] VISIBLE]

http://dx.doi.org/10.4995/ANIAV.2019.8935

Expósito, M. (Ed.) (2010). Nuevos productivismos. Barcelona: MACBA - Servei de Publicacions de la Universitat Autònoma de Barcelona.

Foster, H. (2006). Chat Rooms//2004. En Bishop, C. (ed.), Participation (p. 190-195). London: Whitechapel Gallery. Cambridge, Massachusetts: The MIT Press

Groys, B. (2014). Volverse público. Las transformaciones del arte en el ágora contemporánea. Buenos Aires: Caja Negra Editores.

Groys, B. (2016). Arte en flujo. Ensayos sobre la evanescencia del presente. Buenos Aires: Caja Negra Editores.

Holmes, B. (2008). Investigaciones extradisciplinares. Hacia una nueva crítica de las instituciones. En VVAA. Producción cultural y prácticas instituyetes. Líneas de ruptura en la crítica institucional. Madrid: Traficantes de sueños.

Parramon, R. (2012). Prácticas artísticas, zonas intermedias y utilidad social. En AA.VV., Música para camaleones. El black álbum de la sostenibilidad cultural. Barcelona: Ed. Trànsit Projectes.

Parramon, R. (2018). Estètique de la col·laboració. Intermediacions entre les acciones col·lectives, las participatives i les col·laboratives en les pràctiques artístiques sistèmiques. En Aramburu, N. (Dir.), D'anada i tornada. Projectes i practiques col/laboratives des del museu i a través de l'art. Palma de Mallorca: Fundació Es Baluard Museu d'Art Modern i Contemporani de Palma.

Rancière, J. (2014). El reparto de lo sensible: estética y política. Buenos Aires: Prometeo Libros.

Squibb, S. (2017). Introducción: sobre el modo artístico de producción. En Rosler, M., Clase cultural. Arte y gentrificación. Buenos Aires: Caja Negra.

Zalambani, M. (2018). Borís Arvátov. Teórico del productivismo ruso. En Arvátov, B., Arte y producción (p. 7-40). Madrid: Ediciones Asimétricas.

\section{Referencias de internet:}

Bruguera, T., y Democracia (2011). Arte útil (en línea) http://www.democracia.com.es/comisariado/arte-util--useful-art/, acceso 20 de febrero de 2019.

Kiaer, C. (2009). ¡A la producción!»: los objetos socialistas del constructivismo ruso (en línea)

http://eipcp.net/transversal/0910/kiaer/es, acceso 10 de febrero de 2019.

Mazú, T. (2017). Constructivismo. El arte de producir (en línea) http://www.socialismo-o-barbarie.org/?p=10551, acceso 20 de febrero de 2019.

Rodchenko, A.; Stepánova, V. (1922). Manifiesto productivista (en línea) http://tecnne.com/biblioteca/escritos/manifiestoproductivista, acceso 10 de febrero de 2019. 